\title{
La vacuna anti-Citomegalovirus previene la infección en mujeres de edad fértil
}

\author{
Cytomegalovirus vaccine prevents infection in childbearing age women
}

\section{Objetivo}

Determinar la eficacia y la seguridad de la vacuna recombinante constituida por glicoproteína B de envoltura de Citomegalovirus (CMV) y un nuevo adyuvante (MF59) en una población de mujeres en alto riesgo de contraer la infección.

\section{Diseño}

Ensayo clínico de fase $2^{*}$, aleatorizado, doble ciego y controlado por placebo.

\section{Lugar}

Hospitales de la Universidad de Alabama en Birmingham y Tuscaloosa, Estados Unidos.

\section{Pacientes}

Mujeres ( $n=464)$ de 14 a 40 años, puérperas, seronegativas para anticuerpos anti-CMV, en buen estado de salud y sin enfermedad inmunológica o crónica que requiriese medicación inmunosupresora.

\section{Intervención}

Se aleatorizó a los sujetos en dos grupos para recibir tres dosis de vacuna o placebo $(0,1$ y 6 meses) por vía intramuscular, con rastreo serológico de anticuerpos anti-CMV trimestral durante un seguimiento de 42 meses.

\section{Medición de resultados principales}

Tiempo transcurrido desde la primera dosis de vacuna hasta la detección de infección materna por CMV. El análisis se realizó por intención de tratar.

\section{Resultados principales}

Un análisis intermedio recomendó detener el estudio por la eficacia vacunal. Ver tabla 1. Luego de un mínimo de 12 meses de seguimiento se detectaron 49 infecciones maternas confirmadas y ninguna presentó síntomas durante el período de seroconversión. Se registró una infección congénita en el grupo asignado a vacuna y tres en el grupo placebo, una de ellas con enfermedad sintomática severa, pero no hubo diferencias significativas entre los grupos en las variables relacionadas al embarazo.

Hubo más reacciones locales (dolor, eritema, induración, aumento de la temperatura local) y sistémicas (escalofríos, artralgias y mialgias) en el grupo vacuna que en el grupo placebo.

Tabla 1: efecto de la vacuna anti-citomegalovirus en la incidencia de infección por Citomegalovirus entre los 12 y los 42 meses de seguimiento.

\begin{tabular}{c|c|c|c|c|c}
\hline Resultado & $\begin{array}{c}\text { Grupo Vacuna } \\
\mathbf{n}=\mathbf{2 3 4}\end{array}$ & $\begin{array}{c}\text { Grupo Placebo } \\
\mathbf{n}=\mathbf{2 3 0}\end{array}$ & $\begin{array}{c}\mathbf{N N T} \\
\text { (IC 95\%) }\end{array}$ & $\begin{array}{c}\mathbf{R R R}^{\mathbf{2}} \\
\text { (IC }^{\mathbf{9 5} \%)}\end{array}$ & $\mathbf{p}$ \\
\hline $\begin{array}{c}\text { Infecciones } \\
\text { maternas }\end{array}$ & $18(8 \%)$ & $31(14 \%)$ & $17(9$ a 500$)$ & $50 \%(7$ a 73$)$ & 0,02 \\
\hline
\end{tabular}

"Calculado como densidad de incidencia* cada 100 personas-año.

\section{Conclusiones}

En el grupo que recibió la vacuna glicoproteica B de CMV se reportaron menos casos de infección materna que en el grupo placebo.

Palabras claves: vacuna, prevención, citomegalovirus, ensayo clínico. Keywords: vaccine, prevention, cytomegalovirus, clinical trial. Fuentes de financiamiento: National Institute of Allergy and Infectious Diseases, National Center for Research Resources, Sanofi Pasteur, Chiron (actual Novartis).

\section{Comentario}

La infección congénita por CMV es una causa importante de discapacidad auditiva, cognitiva y motora en recién nacidos. En este sentido y sobre la base de estudios de costo-efectividad ${ }^{1}$, el desarrollo de una vacuna que permita su prevención se considera prioritario en Estados Unidos.

Este estudio de fase 2 presenta la eficacia de una vacuna recombinante para disminuir la incidencia de infección materna en mujeres de alto riesgo, con un perfil de seguridad aceptable. Aunque sería mucho más importante evaluar la eficacia vacunal en términos de disminución de incidencia de infecciones congénitas, el tamaño muestral de este estudio carece del poder necesario para evaluarlo. Por ejemplo, para evaluar como resultado principal a la tasa de infección congénita, se requeriría la inclusión de 3400 mujeres susceptibles (asumiendo una tasa de transmisión de $3 \%$ en el grupo placebo) ó 10.000 (si la tasa fuera del $1 \%)^{2}$. Esto no es fácil de lograr si consideramos que en el presente estudio fueron rastreadas más de 18.000 mujeres para lograr identificar 4509 susceptibles, de las cuales sólo 464 pudieron ser aleatorizadas. Entonces, un estudio que evaluara la incidencia de infección congénita sintomática requeriría más de 50.000 mujeres².

Si bien en este estudio la pérdida de seguimiento de pacientes fue superior al $20 \%$, no hubo diferencias significativas entre ambos grupos. Por otro lado, el intervalo de confianza del resultado primario es amplio, haciendo imprecisa la estimación, y el haber detenido tempranamente el estudio podría llevar a sobreestimar la verdadera eficacia de la vacuna.

\section{Conclusiones de la comentadora}

Se puede considerar que la vacuna presentada en este estudio es un desarrollo prometedor en la búsqueda de una vacuna efectiva contra la infección congénita por CMV, pero aún requiere de estudios de fase $3^{*}$ que avalen su eficacia y seguridad para recomendar su aplicación a nivel poblacional.

Valeria Vietto [ Servicio de Medicina Familiar y Comunitaria. Hospital Italiano de Buenos Aires. valeria.vietto @ hospitalitaliano.org.ar ]

Vietto V. La vacuna anti-Citomegalovirus previene la infección en mujeres en edad fértil. Evid. Actual. Práct. Ambul; 12(3): 85, Jul-Sep 2009. Comentado de: Pass R y col. Vaccine prevention of maternal cytomegalovirus infection. N Engl J Med. 2009;360(12):1191-9. PMID: 19297572. Disponible en URL: http://content.nejm.org/cgi/reprint/360/12/1191.pdf

\section{Referencia}

1. Stratton $\mathrm{K}$ y col. Vaccines for the 21st century: a tool for decision making. Washington, DC: National Academy Press, 2001.

2. Adler S y col. Human CMV vaccine trials: what if CMV caused a rash? J Clin Virol 2008;41:231-236. 